\title{
Deep Neural Network Structure Design for Equipment Failure Prediction in Smart Factory
}

\author{
Sung-Bong Jang ${ }^{1}$ \\ ${ }^{1}$ Associate Professor, Department of Industry-Academy, Kumoh National Institute of Technology, \\ South Korea, sungbong.jang@kumoh.ac.kr
}

\begin{abstract}
A smart factory is a system in which all equipment is connected through the Internet of Things (IoT) and all facilities are operated through a virtual physical system. In the factory, failure prediction for facilities maintenance combined with deep learning has recently attracted much attention to researchers. To perform deep learning-based prediction, a neural network model is built and trained using the learning dataset. The training performance varies depending on the structure of the constructed neural network design. In this study, an efficient design of a neural network is explored that is expected to have the best training performance using a dataset of vibration and temperature. To do this, different neural network models are implemented, and their performance was compared by evaluating loss, accuracy, and execution time. In the experiment, the neural network model was configured to have one input layer, two or three hidden layers, and one output layer, and performance metrics values were measured according to the internal node numbers in every hidden layer. Experimental results show that losses do not increase in spite of that node number becomes large. In general, as the structure of a deep neural network model is complex and the number of nodes inside it increases, it is expected that the loss value will be smaller. For accuracy, there was almost no significant difference between all models. In the case of execution time, it took more time as the number of internal nodes of the model increased.
\end{abstract}

Keywords: Machine Learning, Artificial Neural Network, Deep Learning, Smart Factory

\section{Introduction}

The development of artificial intelligence as well as information and communication technology has brought about rapid changes in the manufacturing sector[1]. As the trend of overproduction and decrease in consumption due to slowing global economic growth becomes evident, the Korean manufacturing industry should take a bigger leap for its survival[2][3]. Recently, the manufacturing fields interest in smart factories has increased in response to these changes. Various techniques have been applied in an attempt to reduce the time and cost invested in facility management[4-6]. Earlier, facility management relied mainly on the experience and intuition of the person in charge. However, the disadvantage of this method is that when the person leaves the company, the accumulated knowhow is not transferred to other employees, resulting in serious technical disconnection and wastage of time and money in facility management[7][8]. In order to solve this problem, an automated predictive maintenance technique is being attempted actively to increase the lifespan of equipment and increase the efficiency of the factory without human intervention. Recently, research on facility maintenance techniques based on deep learning has been very active. Many studies show that deep learning is useful for reducing equipment maintenance costs and increasing accuracy. Most of the research

Received: July 07, 2021; $1^{\text {st }}$ Review Result: August 23, 2021; $2^{\text {nd }}$ Review Result: October 08, 2021 Accepted: November 30, 2021 
focuses on the use of deep learning for equipment error detection, and there are no studies on how to configure the internal structure of the deep neural network after data collection to maximize the error prediction learning performance. However, there is a lack of research on how to construct the structure of a deep neural network for facility processing that enables deep learning to achieve maximum performance. In this study, to alleviate these problems, several deep neural networks have been implemented for deep learning for equipment failure prediction and their performances were compared. The purpose of this study is to obtain hints on the internal structure of deep neural networks that can maximize the predictive performance of equipment faults.

\section{Related Works}

First, Alexandros[9] presents an efficient maintenance (predictive maintenance) method for factory equipment in an Industry 4.0 environment. Whereas previous studies have mainly described from the technical engineering perspective, this study describes the problems and challenges of maintenance of manufacturing equipment from the perspective of cost, digital strategy, and accounting strategy. ChinYi[10] presented a new method for predicting the lifespan of manufacturing equipment. In the past, using an exponential model, it was predicted how long the life of the equipment currently in use would remain or when it would fail. A disadvantage of this model is that it is not efficiently predictable in the event of sudden equipment failures or problems. In the proposed method, the lifespan and failure of equipment were predicted based on time series prediction. In addition, to reflect the characteristics of equipment that suddenly breaks down, a death correlation index value was introduced. In the Jyh-Yih research[11], 2.8 million data collected from 31 wind turbine sensors in Taiwan between 2015 and 2017 were analyzed based on statistical and machine learning techniques and pre-maintained. was used for work. In addition, as machine learning algorithms for predicting failure, decision-tree algorithms and random forest algorithms were used. Z. Weiting[12] describes a data-based predictive maintenance method and suggests a predictive maintenance technique for automatic cleaning equipment in factories. Also, among machine learning algorithms for predictive preservation, artificial neural networks, deep neural networks, and automatic encoder techniques are introduced. Michele[13] describes the limitations, strengths, opportunities, and weaknesses of pre-maintenance of equipment inside a smart factory in an Industry 4.0 environment where all equipment in a factory is interconnected with IoT. Also, among the algorithms for predicting equipment failure in advance, the Markov model, regression analysis, fuzzy-based similarity, and artificial neural network techniques are compared and explained. In addition, research on machine learning techniques that predict equipment failures in factories and minimize maintenance costs by replacing or repairing them in advance is very active. Gian et al.[14] propose a method that is based on machine learning that has a multi-classifier for facilities maintenance. The purpose of the method is to decrease down operating shutdown time and maintenance costs. In the method, they have developed adaptive changing rules to manage facilities status efficiently. Also, the trade-off between sudden breakdown and short equipment lifetime was considered when they design classifiers. To demonstrate the feasibility of the approach, an experiment based on simulation methodology has been conducted, and the results show that their achieves. Changchun[15] propose a facilities maintenance scheme based on a mixed deep neural network that has both long-short term memory (LSTM) and generative adversarial network (GAN). In the scheme, GAN was used to provide a reliable fault dataset to dramatically enhance prediction performance. LSTM is a well-known neural network that is come from recurrent neural network. It is adopted to refer to a long-term data of the previous node in neural network. The advantage of the scheme is that it cannot only inspect facilities status instantly but also prevent sudden fault of the equipment efficiently. Also, they have evaluated the performance in a company located in China by implementing mixed neural network. W. Qi[16] presents a scheme to solve the maintenance problem 
of the railway power facility that is based on LSTM and RNN. In the scheme, LSTM is used to predict a fault of the equipment in advance, and RNN was used to provide sequential dataset to determine most suitable maintenance timing. To conduct an experiment, sample dataset was generated using direct slow down and fault model. The experiment results show that it can achieve the goal of proactive and predictive facilities maintenance.

Unitil now, research on facility maintenance based on deep neural networks has been conducted widely. However, information that describes big data collection and efficient neural network structures is not sufficient. In this study, training data collection for equipment failure prediction as well as deep neural network design for deeper learning is described.

\section{Method}

\subsection{Dataset Processing}

In order to find out the state of the equipment in the smart factory, data on vibration, electricity, temperature, and internal pressure are extracted and used. In most cases, a diagnostic method using vibration data is often used, but it cannot be used for low-speed equipment that does not rotate or for non-vibration equipment[17][18]. Therefore, in the case of electric motors in which a problem occurs due to the flow of electricity rather than vibration, it is more reasonable to diagnose the failure of the equipment by measuring the voltage or current instead of the frequency. Since the equipment in the factory exposes not only one problem but also several problems at the same time, it is necessary to extract data by attaching various types of sensors that can reflect them all. As the number of sensors increases, maintenance costs increase, and data collection and processing methods become very complex. Therefore, when considering cost as the top priority, it is necessary to use the minimum sensor suitable for the characteristics of the equipment.

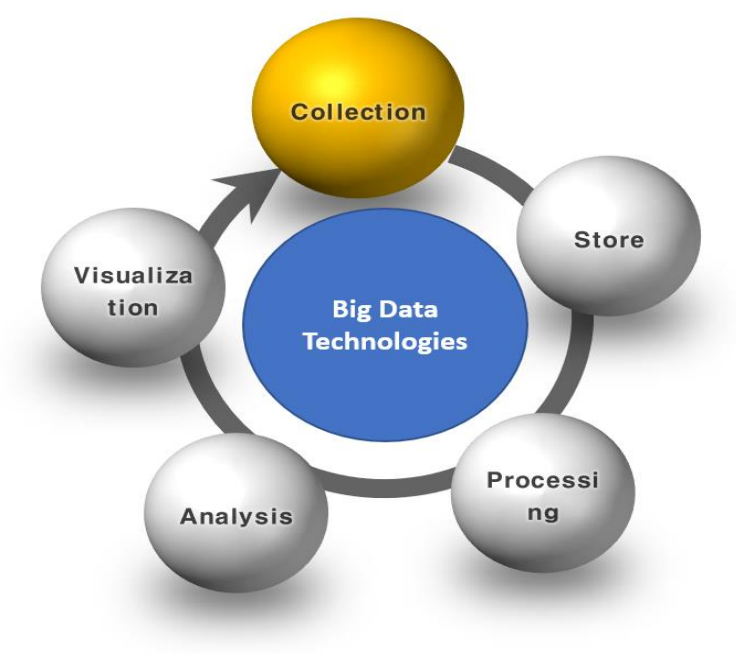

[Fig. 1] Big Data Technologies

Big data generated from facilities are processed through the following steps: firstly, data are collected by various methods such as IoT (e.g., smart sensors, RFID), application programming interface (API), software development kit, and web crawler. Secondly, the collected data must be cleaned as it contains data impurities such as omissions and duplicates. Thirdly, the data are stored after integrating the refined data for data exchange and sharing. With the advent of mobile phones and the Internet after 1990, unstructured data different from the existing data types has exploded, resulting 
in the emergence of the concept of big data. Technologies for processing big data can be divided into collection, storage, processing, analysis, and visualization as shown in [Fig. 1]. Big data can be divided into structured, unstructured, and semi-structured. Structured data refers to data in a fixed format, and an example is data stored in a database. Unstructured data refers to data that does not have a fixed format, such as social data such as Twitter or Facebook. Semi-structured data refers to a form in which the two are mixed. XML or HTML are examples.

\subsection{Data Exploration}

The dataset used includes 29563 rows with vibration, temperatures, and detection labeled with zero or one. In this study, dataset contains lists of columns that store read data. Therefore, if we want to read one column data, we have only to specify its column name. If you look at features, it has nine vibrations and temperatures. The reason for this is because we shift input data to a certain level of number, we can achieve training better performance than simply using only one feature. This dataset is saved in comma-separated value (CSV) format, and it can be read using a software function. Here, pandas library includes data processing functions to make it easier to handle big data for machine learning. The dataset used in this experiment is shown in [Fig. 2].

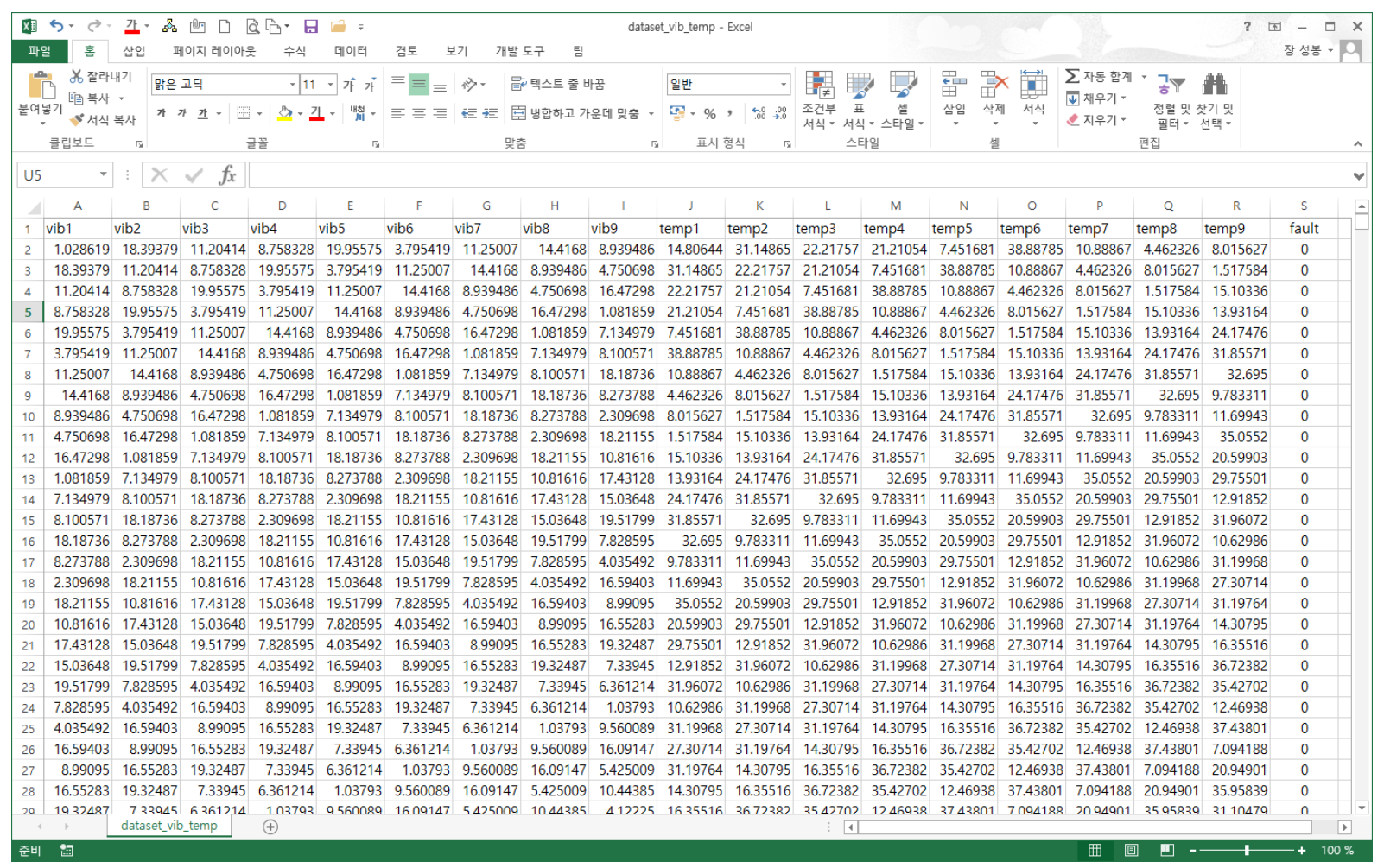

[Fig. 2] Vibration and Temperature Dataset to Train a Defined Deep Neural Network

\subsection{Designing Deep Neural Networks}

In the past, an engineer who is in charge of equipment maintenance visually checks the factory facilities and repairs them if there is a problem. However, in smart factories, a variety of automatic methods can be used to minimize cost and time by predicting failures and taking actions in advance. Recently, an intelligent facility predictive maintenance system based on machine learning has been widely introduced, collecting data such as vibration, temperature, humidity, and noise. Then, a deep 
neural network model is built and trained using the collected data, and failure is predicted using a trained model. In this study, an efficient deep neural network is designed based on the temperature and vibration features of factory facilities. Its proposed structure consists of one input layer, two or three hidden layers, and one output layer. The more the number of nodes in the input layer is, the better, but if there are too many, the training time speed dramatically increases. Here, we have to find the most suitable number of layers and nodes. In our design, the number will be set to be eighteen. The number of nodes in the hidden layer is planned to be between 10 and 800 . As for the output layer, the number of nodes in the layer is set to three because final equipment states have three states of operation, no operation, and failure. [Fig.3] illustrates the proposed architecture.

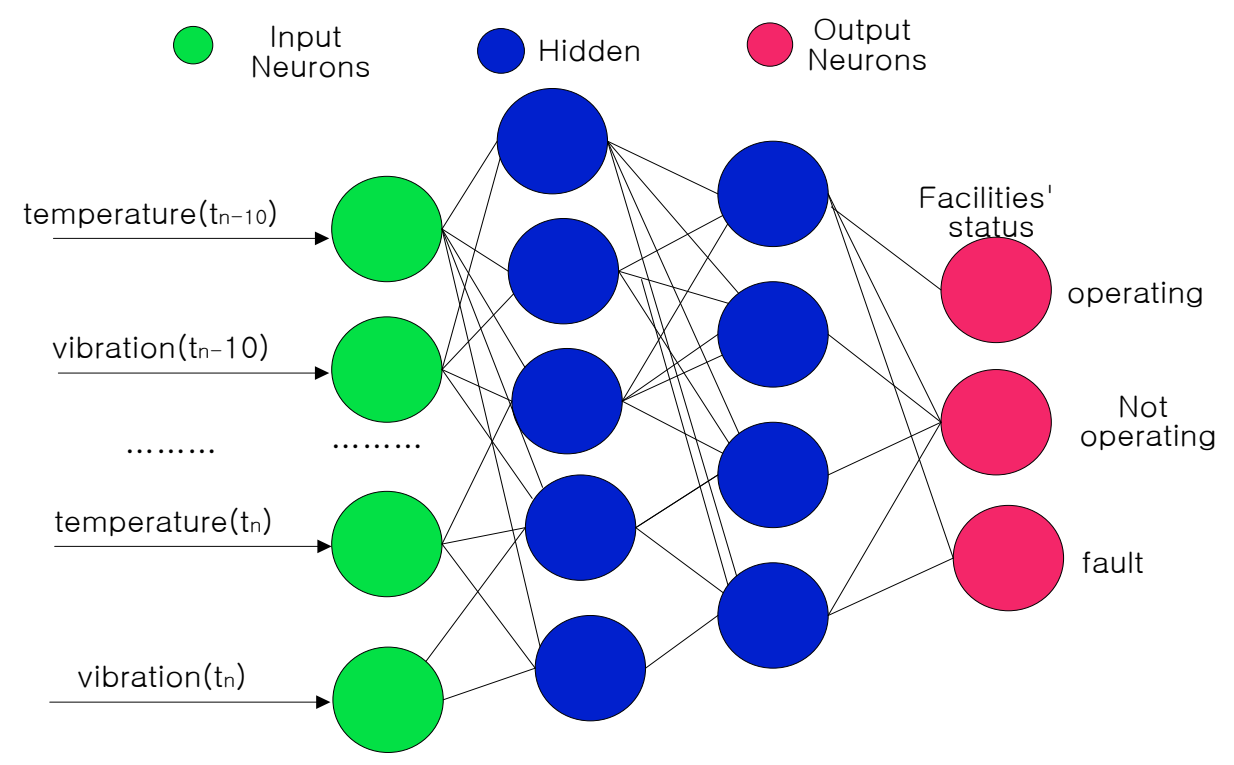

[Fig. 3] Deep Neural Network Structure Design using Vibration and Temperature Features

\subsection{Performance Evaluation Process}

To enable automatic prediction using a defined model, we have to train the model using input dataset. The overall algorithm and processes for failure prediction based on proposed structure is illustrated in [Fig.4].

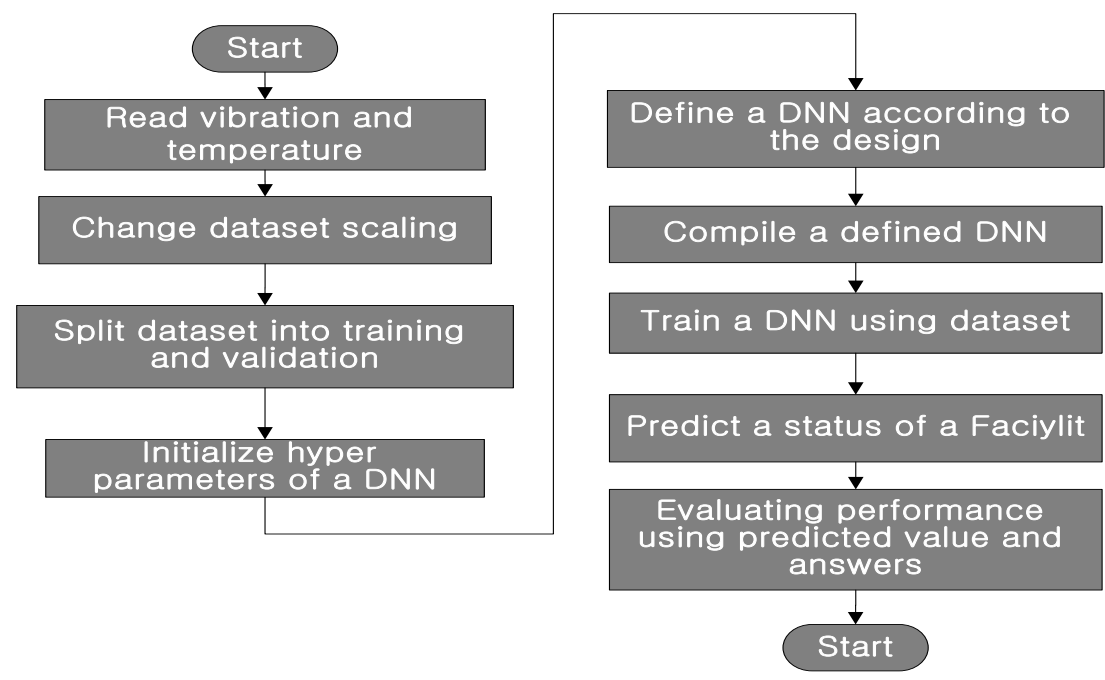

[Fig. 4] Evaluation Processess to Explore a Design of a Deep Neural Network 
The machine learning process for failure prediction was as follows: Firstly, the training data is read. Most of the training data format is saved in Excel or comma separated value (csv) format, and it is read and stored memory as tables. Errors or missing data must be corrected using an appropriate preprocessing technique.

Secondly, the original data values are converted into smaller values by applying a scaling technique so as to minimize the variation caused by large differences among data values. For example, if the vibration is 1000 and the temperature is 0.5 , the difference between the data values will be 999.5 . If we use these data, the training error value becomes too large. In this case, it was standardized using the following formula:

$$
\operatorname{std}(x)=(x-\min (\text { dataset })) /(\max (\text { dataset })-\min (\text { dataset }))
$$

In the above equation, $x$ is the observed value, $\min (\mathrm{A})$ is the minimum value that the attribute (variable) A can have, and $\max (\mathrm{A})$ is the maximum value that the variable $\mathrm{A}$ can have.

Thirdly, the collected equipment-related data was split into training data and verification data. Usually, $80 \%$ of the original data are used as training data, and $20 \%$ as validation data. Also, MinMaxScaler transforms input data to the range set by feature_range. The train_test_split function splits the input data into train and test data. In the function, the variables $\mathrm{x} 1$ and $\mathrm{y} 1$, which store split data, were used as input. The test_size variable is used to specify the split ratio of the input data. In the code, the $\mathrm{x}_{-}$train variable keeps the train data values and $\mathrm{y}_{-}$train stores the target data values. In the same way, $x_{-}$temp stores the test input data, and y_temp stores the target data values for testing.

Fourthly, the hyperparameters are initialized. Hyperparameters include activation function, cost function, initial weights, biases, and learning rates. These are very important for guaranteeing the final performance.

Then, we define the structure of the deep neural network model. In this step, the number of input layers, output layers, and hidden layers are defined, and the number of neurons for each layer is set. The model can be defined using many different structures. The final model was selected after the performance evaluation of each model. In the implementation, we plan to use the sequential function to generate a neural network model. This function was defined in the Keras package. The Keras package provides various functions for defining and handling deep neural networks. If we use this package, it can be implemented more easily. Alternatively, TensorFlow codes can be used to do this. However, the codes are complex and require considerable time to implement.

In the sixth step, the constructed neural network model must be compiled. This step is necessary because we used the TensorFlow tool. If any other tools is used, this step can be skipped.

In the seventh step, machine learning is performed by training the defined model using the input data. At this point, it is necessary to check whether the error rate converges to an appropriate value. If not, the hyperparameters should be adjusted.

In the eighth step, an actual prediction was performed using the trained neural network.

Lastly, after undertaking a performance evaluation on the prediction results, the model with the best performance is selected as the final model. When forecasting is performed later, the equipment condition is predicted using only the selected model.

\section{Results}

This section descrbies experimental results. To explore an efficient design structures for failure detection, an experiment has been conducted where loss and accuracy has been measued after training a defined model. The experimental environments are specified in [Table 1]. 
[Table 1] Experimental Environment

\begin{tabular}{c|c|c}
\hline \multicolumn{2}{c}{ Item } & Specifications for Experiment \\
\hline \multirow{4}{*}{$\begin{array}{c}\text { Environment } \\
\text { Specifications }\end{array}$} & CPU & Intel(R) Core(TM) i7-3770K 3.5 GHz \\
\cline { 2 - 3 } & Memory & 16.0 GB \\
\cline { 2 - 3 } & Operating System & Windows 10 Pro \\
\hline \multirow{4}{*}{$\begin{array}{c}\text { Deep Learning } \\
\text { Parameters }\end{array}$} & Machine Learning Tool & TCDsorflow with Keras \\
\cline { 2 - 3 } & Display & ReLU, sigmoid \\
\cline { 2 - 3 } & Activation Function & 50 \\
\cline { 2 - 3 } & Epoch number & Adam \\
\cline { 2 - 3 } & Optimizer & 5 \\
\hline
\end{tabular}

At first, a model with two hidden layers has been tested. In this test, layer was set as one input layer, two or three hidden layers, and one output layer. Then, losses and accuracies has been measured for various design where node number was set to be different from design to design. The example screen is illustrated in [Fig. 5].

:er factory_equippment_fault Last Checkpoint: 6 minutes ago (autosaved)

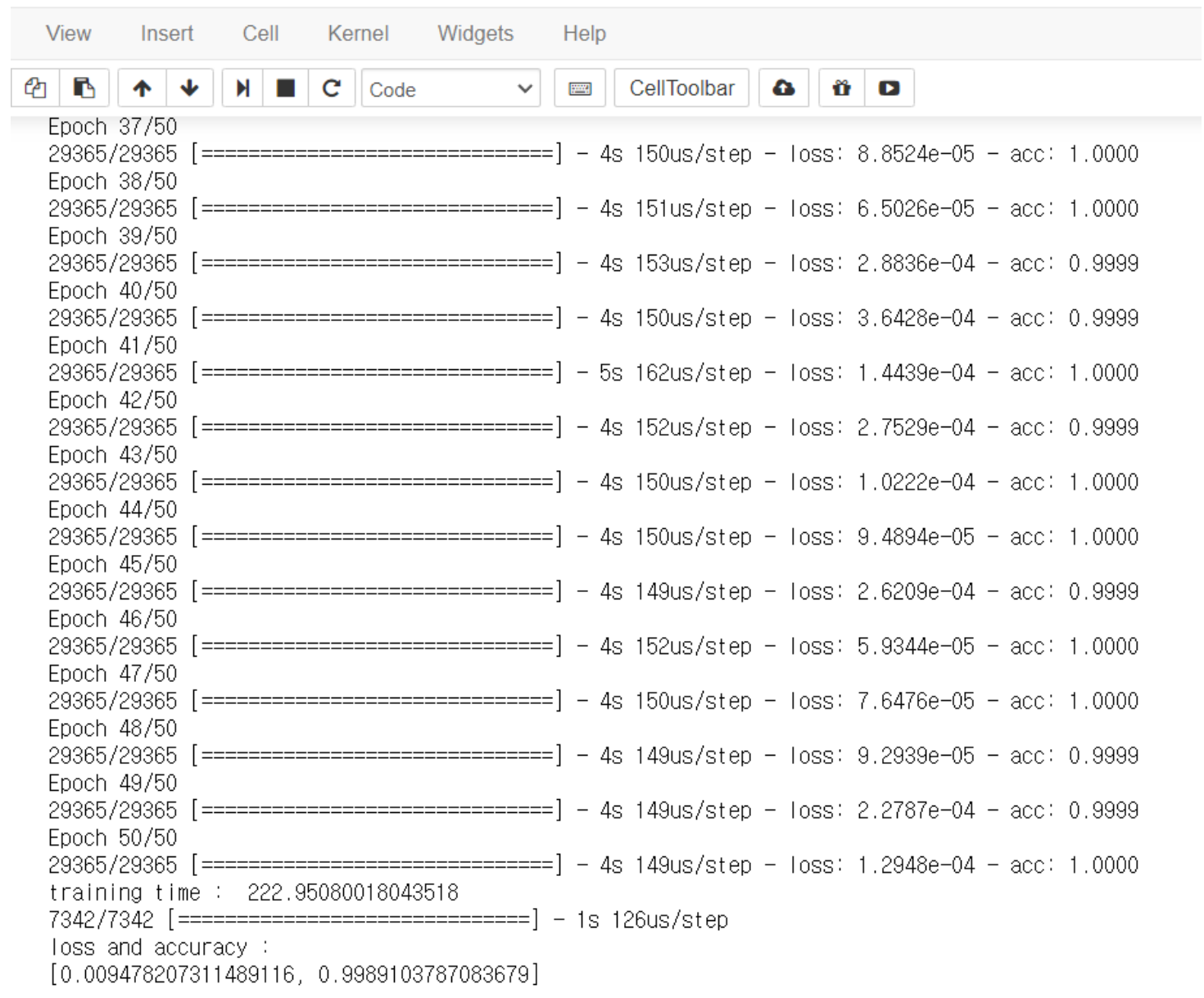

[Fig. 5] Training Example of a Defined Deep Neural Network using Tensorflow

The performance metircs are as follows. First, The root mean square error (RMSE) has been used for metric for measuring losses. It can be calculated using equation (2). 


$$
\text { facult_detection_training_loss }=\sqrt{\frac{1}{n} \sum_{j=1}^{n}\left(P_{j}-R_{j}\right)^{2}}
$$

In equation (2), $n$ is number of data for training deep nueral network, $P_{j}$ represents predicted values, and $R_{j}$ shows real answer values. Sometimes, mean square values without root is used. But, in some cases, it shows too high values. Therefore, we have used RMSE values. Secon, accuracy has been measured. Accuracy shows a difference of error rate of model from 100 percentage. The error rate is the percentage value of the difference of the observed and the actual value, divided by the actual value. This can be calculated using equation (3).

$$
\text { fault_detection_accuracy }=100 \%-\text { Percentage_of_errors }
$$

In equation (3), Percentage_of_errors can be obtained by calculating the percentage of the difference of the predicted value and the answer value, divided by the answer value as shown in equation (4).

$$
\text { percentage_of_error }=\frac{\mid \text { Predicted_Value }- \text { Answer_Value } \mid}{\text { AnswerValue }} \times 100 \%
$$

Third, execution time has been measured to check the performance. The time was calculated by subtracting the time just before the start of the learning from the time after the learning was finished. The experimental results are shown in [Table 2].

\begin{tabular}{|c|c|c|c|c|c|c|c|c|}
\hline \multicolumn{9}{|c|}{ Design with Two Hidden Layers } \\
\hline & $\begin{array}{l}\text { Input } \\
\text { Layer }\end{array}$ & \multicolumn{2}{|c|}{$\begin{array}{c}\text { Hidden Layer } \\
1\end{array}$} & $\begin{array}{l}\text { Hidden } \\
\text { Layer } \\
2\end{array}$ & $\begin{array}{l}\text { Output } \\
\text { Layer }\end{array}$ & Loss & Accuracy & $\begin{array}{l}\text { Execution } \\
\text { Time } \\
\text { (Seconds) }\end{array}$ \\
\hline Model 1 & 18 & \multicolumn{2}{|l|}{10} & 10 & 1 & 0.006686325 & 0.999182784 & 192.83 \\
\hline Model 2 & 18 & \multicolumn{2}{|l|}{25} & 25 & 1 & 0.008696728 & 0.999046581 & 198.41 \\
\hline Model 3 & 18 & \multicolumn{2}{|l|}{50} & 50 & 1 & 0.009478207 & 0.998910378 & 222.95 \\
\hline Model 4 & 18 & \multicolumn{2}{|c|}{100} & 100 & 1 & 0.006652530 & 0.999591392 & 229.68 \\
\hline Model 5 & 18 & \multicolumn{2}{|c|}{200} & 200 & 1 & 0.006643121 & 0.999598062 & 268.90 \\
\hline Model 6 & 18 & \multicolumn{2}{|c|}{400} & 400 & 1 & 0.007664974 & 0.99945518 & 589.37 \\
\hline Model 7 & 18 & \multicolumn{2}{|c|}{800} & 800 & 1 & 0.006644419 & 0.999591392 & 1755.38 \\
\hline \multicolumn{9}{|c|}{ Design with Three Hidden Layers } \\
\hline & $\begin{array}{l}\text { Input } \\
\text { Layer }\end{array}$ & $\begin{array}{l}\text { Hidden } \\
\text { Layer } 1\end{array}$ & $\begin{array}{l}\text { Hidden } \\
\text { Layer } 2\end{array}$ & $\begin{array}{c}\text { Hidden } \\
\text { Layer } \\
3\end{array}$ & $\begin{array}{l}\text { Output } \\
\text { Layer }\end{array}$ & Loss & Accuracy & $\begin{array}{l}\text { Execution } \\
\text { Time } \\
\text { (Seconds) }\end{array}$ \\
\hline Model 8 & 18 & 10 & 10 & 10 & 1 & 0.006801324 & 0.999310596 & 184.09 \\
\hline Model 9 & 18 & 25 & 25 & 25 & 1 & 0.009364327 & 0.999045481 & 188.49 \\
\hline Model 10 & 18 & 50 & 50 & 50 & 1 & 0.008560925 & 0.999181242 & 193.52 \\
\hline Model 11 & 18 & 100 & 100 & 100 & 1 & 0.009324561 & 0.999185438 & 201.87 \\
\hline Model 12 & 18 & 200 & 200 & 200 & 1 & 0.008860928 & 0.999317564 & 270.30 \\
\hline Model 13 & 18 & 400 & 400 & 400 & 1 & 0.006581329 & 0.999599584 & 676.91 \\
\hline
\end{tabular}

[Table 2] Experiment Results of Lossm, Accuracy, and Execution Time for Each Deep Neural Network Design 
In the results, execution time increases a little in propotional to the number of nodes in each design. If epoch number increases a little, execution time increase very much because one epoch is the time to to train whole dataset. For the accuracy results, all designs has almost no difference in that its value is more than $99.9 \%$ (0.999). The reason why the result appears like this is that the number of answer values with one is very small in the dataset. For the loss results, there is almost no difference between a design with two hidden layers and one with three hidden layers. The loss value result is interesting in that model 5 shows the smallest value in a design with two hidden layers. That is, model 5 shows better performance even though it is much less complicated and has smaller internal nodes than those after model 6. From this, we see that it does not increase in spite that node number becomes large. In general, as the structure of a deep neural network model is complex and the number of nodes inside it increases, it is expected that the loss value will be smaller. However, in reality, since meaningless weight values are referenced, the loss value may increase. From designs with three hidden layers, model 13 shows best performance in terms of loss. However, it takes to long time to fininsh training as it is almost 11 minutes.

\section{Conclusions}

In this study, various deep neural network models have been implemented for predicting equipment failures in smart factories, and their performance was compared and analyzed. Loss rate, accuracy, and execution time were used as performance indicators. As a result of the experiment, in terms of loss rate, the model 5 with 200 hidden layer nodes showed the best performance. In terms of accuracy, most models showed similar performance. In the case of execution time, the more complex the model, the longer it took as expected. The future direction of this work is to implement and apply more advanced algorithms such as recurrent neural network or long short-term memory. It is expected that those algorithms shows better performance. The limitations of this study is that a huge AI model was not tested because it takes too long time to finish training. To do this, high-performance computing equipment is necesssary.

\section{Acknowledgments}

This paper was supported by Research Fund, Kumoh National Institute of Technology(2019-104106)

\section{References}

[1] C. Baotong, W. Jiafu, S. Lei, L. Peng, M. Mithun, Y. Boxing Yin, Smart Factory of Industry 4.0: Key Technologies, Application Case, and Challenges, IEEE Access, (2018), Vol.6, pp.6505-6519. DOI: 10.1109/ACCESS.2017. 2783682

[2] S. Devarpita, R. Rajarshi, Reviewing Cyber-Physical System as a Part of Smart Factory in Industry 4.0, IEEE Engineering Management Review, (2020), Vol.48, No.2, pp.103-117, DOI: 10.1109/EMR.2020.2992606

[3] W. Jiafu, C. Baotong, W. Shiyong, X. Min, L. Di, L. Chengliang, Fog Computing for Energy-Aware Load Balancing and Scheduling in Smart Factory, IEEE Transactions on Industrial Informatics, (2018), Vol.14, No.10, pp.4548-4556, DOI: 10.1109/TII.2018.2818932

[4] Z. Tong, T. Dunbing, Z. Haihua, W. Liping, Reinforcement Learning With Composite Rewards for Production Scheduling in a Smart Factory, IEEE Access, (2021), Vol.9, pp.752-766, DOI: 10.1109/ACCESS.2020.3046784

[5] S. Anna, D. Oscar, G. Patrik, Augmented Reality Smart Glasses in the Smart Factory: Product Evaluation Guidelines 
and Review of Available Products, IEEE Access, (2017), Vol.5, pp.9118-9130, DOI: 10.1109/ACCESS.2017. 2703952

[6] F. Giancarlo, M. Fabrizio , R. Domenico, M. L. S. Giuseppe, S. Claudio, A Trust-Based Team Formation Framework for Mobile Intelligence in Smart Factories, IEEE Transactions on Industrial Informatics, (2020), Vol.16, No.9, pp.6133-6142, DOI: 10.1109/TII.2020.2963910

[7] X. Yan, S. Yanming, W. Jiafu, L. Xiaolong, S. Zhiting, Industrial Big Data for Fault Diagnosis: Taxonomy, Review, and Applications, IEEE Access, (2017), Vol.5, pp.17368-17380, DOI: 10.1109/ACCESS.2017.2731945

[8] Y. Jihong, M. Yue, L. Lei, L. Lin, Industrial Big Data in an Industry 4.0 Environment: Challenges, Schemes, and Applications for Predictive Maintenance, IEEE Access, (2017), Vol.5, pp.23484-23491, DOI: 10.1109/ACCESS. 2017.2765544

[9] B. Alexandros, A. Dimitris, M. Gregoris, Predictive Maintenance in the 4th Industrial Revolution: Benefits, Business Opportunities, and Managerial Implications, IEEE Engineering Management Review, (2020), Vol.48, No.1, pp.57-62, DOI: 10.1109/EMR.2019.2958037

[10] L. Chin-Yi, H. Yu-Ming, C. Fan-Tien, H. Hsien-Cheng, A. Muhammad, Time Series Prediction Algorithm for Intelligent Predictive Maintenance, IEEE Robotics and Automation Letters, (2019), Vol.4, No.3, pp.2807-2814, DOI: 10.1109/LRA.2019.2918684

[11] H. Jyh-Yih, W. Yi-Fu, L. Kuan-Cheng, C. Mu-Yen, H. Y. Jenneille, Wind Turbine Fault Diagnosis and Predictive Maintenance Through Statistical Process Control and Machine Learning, IEEE Access, (2020), Vol.8, pp.2342723439, DOI: 10.1109/ACCESS.2020.2968615

[12] Z. Weiting, Y. Dong, W. Hongchao, Data-Driven Methods for Predictive Maintenance of Industrial Equipment: A Survey, IEEE Systems Journal, (2019), Vol.13, No.3, pp.2213-2227, DOI: 10.1109/JSYST.2019.2905565

[13] C. Michele, B. Piero, Z. Enrico, Challenges to IoT-Enabled Predictive Maintenance for Industry 4.0, IEEE Internet of Things Journal, (2020), Vol.7, No.5, pp.4585-4597, DOI: 10.1109/JIOT.2019.2957029

[14] A. S. Gian, S. Andrea, P. Simone, M. Sean, B. Alessandro, Machine Learning for Predictive Maintenance: A Multiple Classifier Approach, IEEE Transactions on Industrial Informatics, (2015), Vol.11, No.3, pp.812-820, DOI: 10.1109/TII.2014.2349359

[15] L. Changchun, T. Dunbing, Z. Haihua, N. Qingwei, A Novel Predictive Maintenance Method Based on Deep Adversarial Learning in the Intelligent Manufacturing System, IEEE Access, (2021), Vol.9, pp.49557-49575, DOI: 10.1109/ACCESS.2021.3069256

[16] W. Qi, B. Siqi, H. Zhengyou, Achieving Predictive and Proactive Maintenance for High-Speed Railway Power Equipment With LSTM-RNN, IEEE Transactions on Industrial Informatics, (2020), Vol.16, No.10, pp.6509-6517, DOI: 10.1109/TII.2020.2966033

[17] S. K. Kahiomba, W. Zenghui, An Effective Predictive Maintenance Framework for Conveyor Motors Using Dual Time-Series Imaging and Convolutional Neural Network in an Industry 4.0 Environment, IEEE Access, (2020), Vol.8, pp.121033-121049, DOI: 10.1109/ACCESS.2020.3006788

[18] M. Michal, W. Maciej, B. Mikollaj, T. Waldemar, K. Tomasz, K. Liliana, Predictive Maintenance of Induction Motors Using Ultra-Low Power Wireless Sensors and Compressed Recurrent Neural Networks, IEEE Access, (2019), Vol.7, pp.178891-178902, DOI: 10.1109/ACCESS.2019.2953019 